\title{
Corrections to "A Further Result about 'on the Channel Capacity of Multiantenna Systems with Nakagami Fading"”
}

\author{
Xianfu Lei, Kai Yu, and Pingzhi Fan \\ Provincial Key Lab of Information Coding and Transmission, Institute of Mobile Communications, Southwest Jiaotong University, \\ Chengdu 610031, China
}

Correspondence should be addressed to Xianfu Lei, xflei81@yahoo.com.cn

Received 15 September 2009; Accepted 21 October 2009

We correct some expressions obtained in the above paper. The corrected expressions will be useful for evaluating the channel capacity of wireless communication systems over fading channels.

Copyright (C) 2009 Xianfu Lei et al. This is an open access article distributed under the Creative Commons Attribution License, which permits unrestricted use, distribution, and reproduction in any medium, provided the original work is properly cited.

\section{Introduction}

In [1], Nadarajah and Kotz have presented some new useful expressions for evaluating the channel capacity of multiantenna systems in Nakagami fading. Here, we would like to correct some expressions in [1].

\section{The Correct Expressions}

We find that [2, equation (2.6.23.4)] is not correctmark and should be modified as (the sign before the function ${ }_{2} F_{2}$ should be "+" instead of "-")

$$
\begin{aligned}
\int_{0}^{\infty} x^{\alpha-1} e^{-p x} \log (a+b x) d x & \\
= & \left(\frac{a}{b}\right)^{\alpha} \frac{\pi}{\alpha \sin \alpha \pi}{ }_{1} F_{1}\left(\alpha ; \alpha+1 ; \frac{a p}{b}\right) \\
-\Gamma(\alpha) p^{-\alpha}\left\{\left[\log \frac{p}{b}-\Psi(\alpha)\right]\right. & \left.\quad+\frac{a p}{b(1-\alpha)}{ }_{2} F_{2}\left(1,1 ; 2,2-\alpha ; \frac{a p}{b}\right)\right\} .
\end{aligned}
$$

Therefore, the expressions in [1] which are derived by using [2, equation (2.6.23.4)] need to be corrected. Specifically, [1, equation (2)] should be modified as

$$
\begin{aligned}
& J(k, \beta)= \frac{2 \pi \beta^{k / 2}}{k \sin (k \pi / 2)}{ }_{1} F_{1}\left(\frac{k}{2} ; 1+\frac{k}{2} ; \beta\right) \\
&-\Gamma\left(\frac{k}{2}\right)\left[\left\{\log \beta-\Psi\left(\frac{k}{2}\right)\right\}\right. \\
& \\
&\left.\quad+\frac{2 \beta}{2-k}{ }_{2} F_{2}\left(1,1 ; 2,2-\frac{k}{2} ; \beta\right)\right] .
\end{aligned}
$$

Similarly, [1, equations (9) and (11)] should be corrected as

$$
\begin{aligned}
J(1, \beta)= & \pi^{3 / 2} \operatorname{erfi}(\sqrt{\beta}) \\
& -\sqrt{\pi}\left[\log \beta+\gamma+2 \log 2+2 \beta_{2} F_{2}\left(1,1 ; 2, \frac{3}{2} ; \beta\right)\right], \\
J(3, \beta)= & -\pi \beta^{1 / 2} \exp (\beta)+\frac{\pi^{3 / 2} \operatorname{erfi}(\sqrt{\beta})}{2} \\
& -\frac{\sqrt{\pi}}{2}\left[\log \beta-2+\gamma+2 \log 2-2 \beta_{2} F_{2}\left(1,1 ; 2, \frac{1}{2} ; \beta\right)\right] .
\end{aligned}
$$

Figure 1 shows the differences between the correct and the wrong expressions. Numerical integration results of [1, equation (1)] are also plotted and corroborate our corrections. The corrections will be helpful for the readers who want to use [2, equation (2.6.23.4)] to evaluate the channel capacity of wireless communication systems over fading channels. 


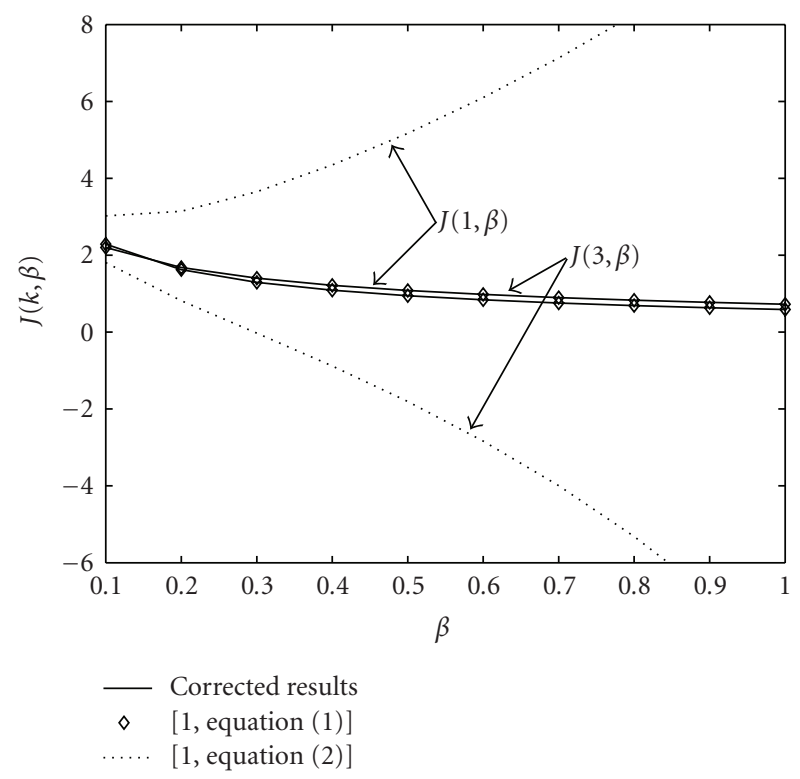

FIGURE 1: Comparisons between the correct and the wrong expressions.

\section{Acknowledgments}

This work was supported by the National 863 HighTech R\&D Program under Grant no. 2009AA01Z238, the 111 Project under Grant no. 111-2-14, and the SinoSwedish Sci \& Tech Cooperation Program under Grant no. 2008DFA12160.

\section{References}

[1] S. Nadarajah and S. Kotz, "A further result about "on the channel capacity of multiantenna systems with Nakagami fading"', EURASIP Journal on Advances in Signal Processing, vol. 2007, Article ID 84713, 2 pages, 2007.

[2] A. P. Prudnikov, Y. A. Brychkov, and O. I. Marichev, Integrals and Series, Vol. 1, Gordon and Breach, Amsterdam, The Netherlands, 1986. 